\title{
Temporal and spatial variation of sea surface temperature, chlorophyll $a$, and primary productivity in the Gulf of California
}

\section{Variación temporal y espacial de temperatura superficial del mar, clorofila $a$ y productividad primaria en el golfo de California}

\author{
Fátima Escalante1, José Eduardo Valdez-Holguín ${ }^{1 *}$, Saúl Álvarez-Borrego ${ }^{2}$, José Rubén Lara-Lara² \\ ${ }^{1}$ Departamento de Investigaciones Científicas y Tecnológicas de la Universidad de Sonora, Blvd. Rosales y \\ Rodríguez s/n, Col. Centro, Hermosillo, CP 83000, Sonora, México. \\ ${ }^{2}$ Centro de Investigación Científica y de Educación Superior de Ensenada (CICESE), División de Oceanología, \\ Carretera Ensenada-Tijuana No. 3918, Zona Playitas, Ensenada, CP 22860, Baja California, México.
}

* Correponding author. E-mail: jvaldez@guayacan.uson.mx

\begin{abstract}
Using satellite derived data from 1997-2010, sea surface temperature (SST), chlorophyll $a$ (Chl) and primary productivity (PP) variations are described at different temporal and spatial scales in the Gulf of California. Interannual signals of El Niño (EN) and La Niña (LN) were more evident at the entrance to the gulf. Once EN or LN events were over, there was a delayed response of three to six months for the reestablishment of "normal" phytoplankton activity levels. Phytoplankton response to high concentrations of nutrients due to LN was not immediate. At regional scale, EN significantly decreased the Chl mean with regard to "normal" conditions at the entrance, central gulf, and Midriff Islands region (MIR); however, PP under EN conditions decreased significantly only at the entrance. The increase in Chl and PP due to LN relative to "normal" conditions was significant only at the entrance. SST, Chl, and PP annual signals are the main source of variation, particularly for the central gulf and entrance, but in the MIR, "winter" PP $\left(1.96 \mathrm{~g} \mathrm{C} \mathrm{m}^{-2} \mathrm{~d}^{-1}\right)$ was not significantly different from that of "summer" $\left(1.62 \mathrm{~g} \mathrm{C} \mathrm{m}^{-2} \mathrm{~d}^{-1}\right)$. Highest PP was found in the central gulf and MIR, where inferences of production attributable to new production were estimated at more than $60 \%$ of PP. At the gulf entrance, primary productivity was mainly attributable to regenerated production, particularly during summer and autumn.
\end{abstract}

Key words: Gulf of California, chlorophyll $a$, primary productivity, remote sensing, El Niño.

RESUMEN. Con datos satelitales de 1997 a 2010, se describe la variación de temperatura superficial del mar (TSM), clorofila a (Chl) y productividad primaria (PP) a diferentes escalas temporales y espaciales en el golfo de California. Las señales interanuales El Niño (EN) y La Niña (LN) fueron más evidentes en la boca del golfo. Existió una respuesta desfasada de tres a seis meses para el restablecimiento de las condiciones "normales" de la actividad fitoplanctónica una vez que cesaba un evento EN o LN. La respuesta fitoplanctónica ante las mayores concentraciones de nutrientes que supone el evento LN no fue inmediata. En la escala regional, la condición EN redujo significativamente el promedio de Chl respecto a condiciones "normales" en la boca, el centro y la región de las islas grandes (RIG) del golfo; sin embargo, la PP bajo la condición EN sólo se redujo significativamente en la boca. El incremento de Chl y PP por LN con relación a condiciones "normales" sólo fue significativo en la boca. Se confirma que la señal anual de TSM, Chl y PP es la componente mayor de la variación, sobre todo para las regiones de la boca y centro. Sin embargo, en la RIG, la PP de "invierno" $\left(1.96 \mathrm{~g} \mathrm{C} \mathrm{m}^{-2} \mathrm{~d}^{-1}\right)$ no fue significativamente diferente a la de "verano" $\left(1.62 \mathrm{~g} \mathrm{C} \mathrm{m}^{-2} \mathrm{~d}^{-1}\right)$. La PP más alta se encontró en la RIG y en el centro del golfo, en donde las inferencias de producción atribuible a la producción nueva se calcularon en más del $60 \%$ de PP. En la boca del golfo, la productividad primaria fue principalmente atribuible a la producción regenerada, particularmente durante verano y otoño.

Palabras clave: golfo de California, clorofila $a$, productividad primaria, percepción remota, El Niño.

\section{INTRODUCTION}

The Gulf of California is one of the most productive oceanic ecosystems $\left(>300 \mathrm{~g} \mathrm{C} \mathrm{m}^{-2} \mathrm{yr}^{-1}\right)$ with marked seasonal and interannual variation (Álvarez-Borrego and Lara-Lara 1991, Álvarez-Borrego 2010). The most prominent interannual signal in the region is produced by the sequence of the El Niño (EN) and La Niña (LN) phenomena (Soto-Mardones et al. 1999), which impact phytoplankton biomass (Lara-Lara et al. 1984, Valdez-Holguín and Lara-Lara 1987, Santamaríadel-Ángel et al. 1994a). During EN, there is an increase in

\section{INTRODUCCIÓN}

El golfo de California es uno de los ecosistemas oceánicos más productivos $\left(>300 \mathrm{~g} \mathrm{C} \mathrm{m}^{-2}\right.$ año $\left.{ }^{-1}\right)$ en el que existe una variación estacional e interanual claramente marcada (Álvarez-Borrego y Lara-Lara 1991, ÁlvarezBorrego 2010). La señal interanual más prominente en la región proviene de la secuencia de los fenómenos climatológicos El Niño (EN) y La Niña (LN) (Soto-Mardones et al. 1999), los cuales impactan a la biomasa fitoplanctónica (Lara-Lara et al. 1984, Valdez-Holguín y Lara-Lara 1987, 
sea surface temperature (SST) (Soto-Mardones et al. 1999, Lavín et al. 2003) associated with a decrease in nutrient and chlorophyll $a(\mathrm{Chl})$ concentrations and primary productivity (PP) (Santamaría-del-Ángel et al. 1994a, 1994b; EspinosaCarreón and Valdez-Holguín 2007). The sinking of the thermocline and the occurrence of coastal upwelling, which transports relatively warm and nutrient-poor waters to the surface during EN (Dajoz 2002), limit phytoplankton growth. Conversely, the decrease in SST, relatively low sea level, and rising of the thermocline associated with $\mathrm{LN}$ conditions increase nutrient and PP levels (Dajoz 2002).

The Gulf of California acts as an evaporation basin that gains heat through its surface (Lavín and Organista 1988), with seasonal modulation (Ripa and Marinone 1989). The wind regime is variable: northwesterly winds prevail from November to May and southeasterly winds predominate the rest of the year, bringing humid air and tropical climate conditions (Merrifield and Winant 1989). Northwesterly winds generate upwelling that promotes nutrient enrichment of the euphotic zone and thus phytoplankton growth and PP (Zeitzschel 1969); Chl values greater than $10 \mathrm{mg} \mathrm{m}^{-3}$ have been recorded in winter and spring (Álvarez-Borrego and Lara-Lara 1991, Álvarez-Borrego 2010). In summer and autumn, strong upwelling (on the peninsular side) does not occur due to strong thermal stratification and its effect on phytoplankton biomass is weak (Santamaría-del-Ángel et al. 1999). Intense tide-related mixing is responsible for the high productivity that characterizes the region of the two large Midriff Islands, Tiburón and Ángel de la Guarda (ÁlvarezBorrego and Lara-Lara 1991). At the mouth of the gulf, the oceanographic conditions are similar to those of the East Pacific Ocean due to free water exchange (Álvarez-Borrego 1983).

The seasonal variability in the vertical structure of the Gulf of California causes the mixed-layer depth to increase from summer to winter and stimulates the vertical diffusion of nitrate $\left(\mathrm{NO}_{3}^{-}\right)$and other nutrients to the euphotic zone. Hence, PP is higher in winter and spring, and lower in summer when surface waters are warmer, stratified, and oligotrophic (Hidalgo-González and Álvarez-Borrego 2004). White et al. (2007) concluded that nitrogen fixation carried out by diazotrophs, which convert atmospheric nitrogen $\left(\mathrm{N}_{2}\right)$ into ammonium $\left(\mathrm{NH}_{4}{ }^{+}\right)$, supplied 35-48\% of the phytoplankton nitrogen demand during summer in the central basins (Guaymas and Carmen) of the Gulf of California, even though a considerable percentage of summertime PP is regenerated production.

Net water exchange between the Pacific Ocean and Gulf of California, with outflow of surface water $(0-200 \mathrm{~m})$ and inflow of deep water (200-600 m), results in a net input of $\mathrm{NO}_{3}{ }^{-}$and other nutrients to the gulf, which are carried to the euphotic zone by upwelling and mixing year-round in the Midriff Islands region (MIR) and throughout the gulf in winter and spring (Álvarez-Borrego 2010). HidalgoGonzález and Álvarez-Borrego (2004) indicated that surface
Santamaría-del-Ángel et al. 1994a). Durante EN se registró un incremento de la temperatura superficial del mar (TSM) (Soto-Mardones et al. 1999, Lavín et al. 2003) asociado a un decremento de la concentración de nutrientes y de clorofila $a$ (Chl) y la productividad primaria (PP) (Santamaría-delÁngel et al. 1994a, 1994b; Espinosa-Carreón y ValdezHolguín 2007). El hundimiento de la termoclina y la presencia de surgencias costeras, que acarrean a la superficie aguas relativamente cálidas y pobres en nutrientes durante EN (Dajoz 2002), limitan el crecimiento del fitoplancton. Por el contrario, el decremento de la TSM, el nivel del mar relativamente bajo y el levantamiento de la termoclina, propiciados por condiciones LN, incrementan la concentración de nutrientes y los niveles de PP (Dajoz 2002).

El golfo de California actúa como una cuenca de evaporación que gana calor a través de su superficie (Lavín y Organista 1988), con una modulación estacional (Ripa y Marinone 1989). El régimen de vientos dentro del golfo es variable: de noviembre a mayo prevalecen vientos provenientes del noroeste, el resto del año prevalecen vientos procedentes del sureste que introducen aire húmedo y producen condiciones climáticas de tipo tropical (Merrifield y Winant 1989). Los vientos del noroeste generan surgencias que incrementan los nutrientes en la zona eufótica y, por lo tanto, promueven el crecimiento de fitoplancton y el aumento de PP (Zeitzschel 1969); se han registrado valores de Chl por encima de $10 \mathrm{mg} \mathrm{m}^{-3}$ durante invierno y primavera (ÁlvarezBorrego y Lara-Lara 1991, Álvarez-Borrego 2010). En verano y otoño, no hay surgencias fuertes del lado peninsular del golfo debido a la fuerte estratificación térmica, por lo que su efecto en la biomasa de fitoplancton es débil (SantamaríaDel-Ángel et al. 1999). La mezcla intensa causada principalmente por fenómenos asociados a las mareas es responsable de la alta productividad que caracteriza la región de las islas grandes, Tiburón y Ángel de la Guarda (Álvarez-Borrego y Lara-Lara 1991). En la boca del golfo, las condiciones oceanográficas son similares a las del océano Pacifico oriental debido al libre intercambio de agua (Álvarez-Borrego 1983).

La variabilidad estacional de la estructura vertical del golfo de California determina que la profundidad de la capa de mezcla aumente de verano a invierno y se promueva la difusión vertical de nitrato $\left(\mathrm{NO}_{3}^{-}\right)$y otros nutrientes a la zona eufótica. Por tal razón, la PP es mayor en invierno y primavera, y menor en verano, cuando el agua superficial es cálida, está estratificada y es oligotrófica (Hidalgo-González y Álvarez-Borrego 2004). White et al. (2007) concluyeron que la demanda fitoplanctónica de nitrógeno durante el verano en las cuencas centrales del golfo de California (Guaymas y Carmen) es provista en un $35-48 \%$ por la fijación de nitrógeno realizada por organismos diazótrofos, que transforman el nitrógeno atmosférico $\left(\mathrm{N}_{2}\right)$ en amonio $\left(\mathrm{NH}_{4}{ }^{+}\right)$, a pesar de que un porcentaje considerable de la PP durante el verano es producción regenerada. 
$\mathrm{NO}_{3}^{-}$increases during cold conditions (e.g., values of $<0.5 \mu \mathrm{M}$ at the entrance, $\sim 0.5-1.0 \mu \mathrm{M}$ in the central gulf, and $\sim 7.0 \mu \mathrm{M}$ in the MIR, at $0-20 \mathrm{~m}$ depth) and decreases under warm conditions (e.g., values of $<1.0 \mu \mathrm{M}$ for the entrance and central gulf, and $\sim 3.5 \mu \mathrm{M}$ for the MIR, at $0-20 \mathrm{~m}$ depth). The MIR, where the nutricline is shallower, has the highest mean surface nitrate values (Hidalgo-González and ÁlvarezBorrego 2004).

It has been suggested that under certain wind regimes, some gulf regions, such as the mouth, are more susceptible to phenomena like EN overlapping the effects of interannual climate events and regional seasonal conditions (Beier and Ripa 1998, Carrillo et al. 2002, Soto-Mardones et al. 1999).

The objective of the present work was to describe the spatial and temporal variability of SST, Chl, and PP in the Gulf of California in order to determine the interannual and seasonal conditions under which $\mathrm{Chl}$ and $\mathrm{PP}$ attain maximum and minimum levels in the MIR, central gulf, and entrance. A statistical analysis is presented of the overlapping of the interannual and seasonal conditions of SST, Chl, and PP in the Gulf of California estimated from remote sensing data. Information reported in previous studies is updated with information available to date.

\section{MATERIALS AND METHODS}

The SST $\left({ }^{\circ} \mathrm{C}\right)$, Chl $\left(\mathrm{mg} \mathrm{m}^{-3}\right)$, and PP $\left(\mathrm{g} \mathrm{C} \mathrm{m}^{-2} \mathrm{~d}^{-1}\right)$ data for the period 1997-2010 were obtained from the OceanColor Web and OceanWatch North Pacific Demonstration Project (OWNPDP). SST was derived from MODIS, AVHRR, and a combination of sensors labeled Blended Sea Surface Temperature by OWNPDP. Chl was obtained from SeaWiFS and MODIS. Data were not reprocessed nor was any compensatory adjustment from different sensors used; they were used, without combining, to complete the series The PP data were not reprocessed because they are processed by NASA using the vertically generalized production model (VGPM) proposed by Behrenfeld and Falkowski (1997):

$$
\operatorname{PP}\left(\mathrm{mg} \mathrm{C} \mathrm{m}^{-2} \mathrm{~d}^{-1}\right)=\mathrm{P}_{\text {opt }} \mathrm{f}\left(\mathrm{PAR}_{\mathrm{o}}\right) \mathrm{DL} \mathrm{Chl}_{\text {sat }} \mathrm{Z}_{\text {eu }}
$$

where $\mathrm{P}_{\text {opt }}^{\mathrm{b}}$ is the photosynthetic ratio (photosynthesis per unit of $\mathrm{Chl}$ ) at the optimum light level in the water column $\left(\mathrm{mgC} \mathrm{mgChl}^{-1} \mathrm{~h}^{-1}\right), \mathrm{f}\left(\mathrm{PAR}_{\mathrm{o}}\right)$ is a function that represents the effect of light in all the euphotic zone and throughout the day, DL is the length of day in hours (according to latitude and date), and $Z_{\text {eu }}$ is the depth of the euphotic zone (m) calculated from Chl following Morel and Berthon (1989). The VGPM is a non-spectral, homogeneous biomass vertical distribution, vertically integrated production model. The main source of variability of these estimates is given by $\mathrm{P}^{\mathrm{b}}{ }_{\mathrm{opt}}$; exploring different values of this parameter is beyond the scope of this paper.
El intercambio neto de agua entre el océano Pacífico y el golfo de California, salida de agua superficial $(0-200 \mathrm{~m})$ y entrada de agua profunda (200-600 m), resulta en un aporte neto de $\mathrm{NO}_{3}^{-}$y otros nutrientes hacia el golfo, mismos que son acarreados a la zona eufótica por las surgencias y la mezcla en todo el golfo en invierno y primavera y en la región de las islas grandes (RIG) en todo el año (ÁlvarezBorrego 2010). Hidalgo-González y Álvarez-Borrego (2004) indicaron que el $\mathrm{NO}_{3}{ }^{-}$superficial se incrementa durante condiciones frías (e.g., valores de $<0.5 \mu \mathrm{M}$ en la boca, $\sim 0.5-1.0 \mu \mathrm{M}$ en el centro y $\sim 7.0 \mu \mathrm{M}$ en la RIG, a $0-20 \mathrm{~m}$ de profundidad) y disminuye con condiciones cálidas (e.g., valor de $<1.0 \mu \mathrm{M}$ en la boca y el centro del golfo y $\sim 3.5 \mu \mathrm{M}$ en la RIG, a 0-20 m de profundidad). En la RIG, la nutriclina es más superficial y promedia los valores de nitrato superficial más elevados (Hidalgo-González y Álvarez-Borrego 2004).

Se ha sugerido que, bajo ciertos regímenes de viento, algunas regiones del golfo, como la boca, son más susceptibles a fenómenos como EN, entrecruzando los efectos de eventos climatológicos interanuales con las condiciones estacionales regionales (Beier y Ripa 1998, Carrillo et al. 2002, Soto-Mardones et al. 1999).

El objetivo del presente trabajo fue describir la variabilidad espaciotemporal de la TSM, Chl y PP en el golfo de California, a fin de determinar las condiciones interanuales y estacionales bajo las cuales la Chl y PP alcanzan los valores máximos y mínimos en la RIG, el centro y la boca del golfo de California. En este trabajo se presenta un análisis estadístico del entrecruzamiento entre las condiciones interanuales y estacionales de TSM, Chl y $\mathrm{PP}$ en el golfo de California estimadas a partir de sensores remotos. Se actualiza la información de estudios previos con la información disponible hasta la fecha de estudio.

\section{MATERIALES Y MÉTODOS}

Los datos de TSM $\left({ }^{\circ} \mathrm{C}\right)$, Chl $\left(\mathrm{mg} \mathrm{m}^{-3}\right)$ y PP $\left(\mathrm{g} \mathrm{C} \mathrm{m}^{-2} \mathrm{~d}^{-1}\right)$ fueron obtenidos para el periodo 1997-2010 a través de OceanColorWeb y Ocean Watch North Pacific Demonstration Project (OWNPDP). La TSM se obtuvo de MODIS, AVHRR y de una combinación de sensores etiquetados por OWNPDP como Blended Sea Surface Temperature. La Chl se obtuvo de SeaWiFS y MODIS. Los datos no fueron reprocesados ni se utilizó ningún ajuste compensatorio proveniente de distintos sensores; los datos fueron usados para completar la serie, sin haberse combinado entre ellos. Los datos de PP tampoco fueron reprocesados, ya que son el resultado del procesamiento que hace la NASA empleando el modelo de producción verticalmente generalizado (VGPM, por sus siglas en inglés) de Behrenfeld y Falkowski (1997):

$$
\operatorname{PP}\left(\mathrm{mg} \mathrm{C} \mathrm{m}^{-2} \mathrm{~d}^{-1}\right)=\mathrm{P}_{\text {opt }}^{\mathrm{b}} \mathrm{f}\left(\mathrm{PAR}_{\mathrm{o}}\right) \mathrm{DL} \mathrm{Chl}_{\mathrm{sat}} \mathrm{Z}_{\mathrm{eu}}
$$


Data were downloaded as monthly composites and/or daily images (clear and complete), and monthly matrices were generated with the Beam-VISAT software. Data were processed and analyzed statistically at regional scale (fig. 1; MIR, central gulf, and entrance) and overall (considering the gulf as a unit), and at seasonal, annual, and interannual time scales.

Analysis of variance (ANOVA) was performed followed by the Tukey-Kramer test ( $95 \%$ confidence level) to determine differences between seasons and EN, LN, and "normal" hydrographic conditions, and between regions. Seasonal anomalies were calculated (mean seasonal value minus the mean for all the study period) in order to infer the duration of the effects of EN and LN events on Chl and PP.

Total PP of an ecosystem consists of regenerated production $\left(\mathrm{P}_{\mathrm{R}}\right)$, based on the reutilization of reduced nitrogen resulting from respiration in the euphotic zone, plus new production $\left(\mathrm{P}_{\mathrm{N}}\right)$, based on the input of new nutrients (oxidized nitrogen) (Dugdale and Goering 1967). Considering that the lowest total PP values estimated for the Gulf of California range from 0.36 to $0.43 \mathrm{~g} \mathrm{C} \mathrm{m}^{-2} \mathrm{~d}^{-1}$ in summer (HidalgoGonzález and Álvarez-Borrego 2001) and that White et al.

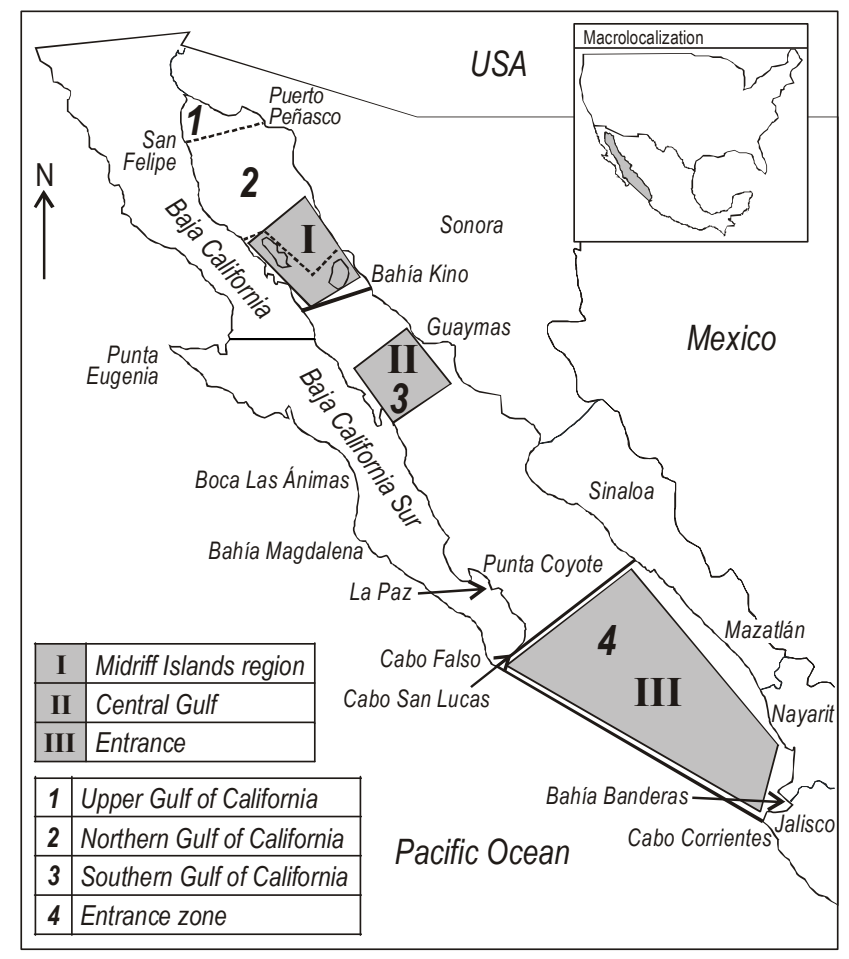

Figure 1. Gulf of California. Roman numerals indicate the regions surveyed. Arabic numerals indicate the regionalization made by Lavín and Marinone (2003) (modified from Lavín and Marinone 2003).

Figura 1. Golfo de California. En números romanos se señalan las regiones de muestreo de este trabajo. En números arábigos se señala la regionalización hecha por Lavín y Marinone (2003) (modificado de Lavín y Marinone 2003). donde $\mathrm{P}^{\mathrm{b}}{ }_{\mathrm{opt}}$ es la razón fotosintética (fotosíntesis por unidad de $\mathrm{Chl}$ ) en el óptimo de luz en la columna de agua ( $\mathrm{mgC}$ $\left.\mathrm{mgCh}^{-1} \mathrm{~h}^{-1}\right), \mathrm{f}\left(\mathrm{PAR}_{\mathrm{o}}\right)$ es una función que representa el efecto de la luz en toda la zona eufótica y a través de todo el día, DL es la longitud del día en horas (de acuerdo con la latitud y fecha) y $Z_{\text {eu }}$ es la profundidad de la zona eufótica (m) calculada con Chl de acuerdo con Morel y Berthon (1989). El VGPM es un modelo de producción no espectral, con distribución vertical homogénea de biomasa fitoplanctónica, e integrado verticalmente. La principal fuente de variabilidad de estas estimaciones está dada por el parámetro $\mathrm{P}^{\mathrm{b}}{ }_{\mathrm{opt}}$; explorar diferentes valores de este parámetro esta fuera de los objetivos del trabajo.

Los datos se descargaron como composiciones mensuales y/o imágenes diarias (claras y completas), a partir de las cuales se generaron las matrices mensuales con el programa Beam-VISAT. Los datos fueron procesados y analizados estadísticamente a escala espacial-regional (fig. 1; RIG, centro y boca del golfo) y global (considerando al golfo como una unidad)-y temporal (estacional, anual e interanual).

Se realizaron análisis de varianza (ANOVA) seguidos de la prueba Tukey-Kramer (95\% de nivel de confianza) para probar las hipótesis sobre diferencias entre estaciones y condiciones hidrográficas EN, LN y "normal", y entre regiones. Se calcularon anomalías estacionales (el valor promedio de cada estación menos el promedio de todo el período de estudio) a fin de inferir la duración de los efectos de eventos EN y LN sobre la Chl y PP.

La PP total de un ecosistema está compuesta de la producción regenerada $\left(\mathrm{P}_{\mathrm{R}}\right)$, basada en la reutilización del nitrógeno reducido, producto de la respiración en la zona eufótica, más la producción nueva $\left(\mathrm{P}_{\mathrm{N}}\right)$, basada en la entrada de nutrientes nuevos (nitrógeno oxidado) (Dugdale y Goering 1967). Considerando que en el golfo de California los niveles estimados de productividad total más bajos fueron entre 0.36 a $0.43 \mathrm{~g} \mathrm{C} \mathrm{m}^{-2} \mathrm{~d}^{-1}$ durante verano (Hidalgo-González y Álvarez-Borrego 2001) y que White et al. (2007) establecen que bajo esas condiciones la producción básicamente se debe a la producción regenerada, se supone como primera aproximación a la realidad que cualquier valor por encima de esta PP de verano es atribuible a la $P_{N}$.

\section{RESUlTADOS}

Los promedios anuales de Chl y PP presentaron un gradiente a lo largo del golfo de California, con características oligotróficas en la boca $\left(0.46 \mathrm{mg} \mathrm{m}^{-3}, 0.92 \mathrm{~g} \mathrm{C} \mathrm{m}^{-2} \mathrm{~d}^{-1}\right)$, mesotróficas en la región centro $\left(0.83 \mathrm{mg} \mathrm{m}^{-3}, 1.52 \mathrm{~g} \mathrm{C} \mathrm{m}^{-2}\right.$ $\left.\mathrm{d}^{-1}\right)$ y eutróficas en la RIG $\left(1.44 \mathrm{mg} \mathrm{m}^{-3}, 2.14 \mathrm{~g} \mathrm{C} \mathrm{m}^{-2} \mathrm{~d}^{-1}\right)$ (tabla 1). El promedio anual de TSM fue más alto en la boca $\left(25.57^{\circ} \mathrm{C}\right)$, intermedio en la región central $\left(24.63{ }^{\circ} \mathrm{C}\right)$ y más bajo en la RIG $\left(23.24^{\circ} \mathrm{C}\right)$. Los promedios anuales de TSM, Chl y PP para todo el golfo fueron $24.48{ }^{\circ} \mathrm{C}, 0.91 \mathrm{mg} \mathrm{m}^{-3}$ y $1.52 \mathrm{~g} \mathrm{C} \mathrm{m}^{-2} \mathrm{~d}^{-1}$, respectivamente (tabla 1). Para las 
Escalante et al.: Primary production in the Gulf of California

(2007) established that under those conditions production is mainly attributed to $\mathrm{P}_{R}$, it can be assumed, as a first approximation to reality, that any value above this summertime PP is attributed to $\mathrm{P}_{\mathrm{N}}$.

\section{RESUlTS}

The mean Chl and PP values presented a gradient along the Gulf of California, with oligotrophic characteristics at the entrance $\left(0.46 \mathrm{mg} \mathrm{m}^{-3}, 0.92 \mathrm{~g} \mathrm{C} \mathrm{m}^{-2} \mathrm{~d}^{-1}\right)$, mesotrophic conditions in the central gulf $\left(0.83 \mathrm{mg} \mathrm{m}^{-3}, 1.52 \mathrm{~g} \mathrm{C} \mathrm{m}^{-2} \mathrm{~d}^{-1}\right)$, and eutrophic conditions in the MIR $\left(1.44 \mathrm{mg} \mathrm{m}^{-3}, 2.14 \mathrm{~g} \mathrm{C} \mathrm{m}^{-2}\right.$ $\mathrm{d}^{-1}$ ) (table 1). Mean annual SST was highest at the entrance $\left(25.57^{\circ} \mathrm{C}\right)$, intermediate in the central gulf $\left(24.63{ }^{\circ} \mathrm{C}\right)$, and lowest in the MIR $\left(23.24^{\circ} \mathrm{C}\right)$. The mean annual SST, Chl, and $\mathrm{PP}$ values for the entire gulf were $24.48^{\circ} \mathrm{C}, 0.91 \mathrm{mg} \mathrm{m}^{-3}$, and $1.52 \mathrm{~g} \mathrm{C} \mathrm{m}^{-2} \mathrm{~d}^{-1}$, respectively (table 1 ). For the comparisons of the mean values of the variables under consideration, among regions and among conditions, the ANOVA results were confirmed in all cases by the Tukey-Kramer tests, so only the former will be presented. Mean Chl values for the entire gulf during EN $\left(0.74 \mathrm{mg} \mathrm{m}^{-3}\right)$ and $\mathrm{LN}\left(1.00 \mathrm{mg} \mathrm{m}^{-3}\right)$ events were statistically different $\left(F_{(2,468)}=7.22, P=0.000\right)$ from the "normal" conditions (SST anomalies close to zero). Mean Chl for the entire gulf decreased 23\% during EN and increased 4\% during LN relative to "normal" conditions. Mean SST and PP values for the entire gulf showed significant differences between EN $\left(25.50{ }^{\circ} \mathrm{C}, 1.45 \mathrm{~g} \mathrm{C} \mathrm{m}^{-2} \mathrm{~d}^{-1}\right)$ and $\mathrm{LN}\left(24.19^{\circ} \mathrm{C}, 1.50 \mathrm{~g} \mathrm{C} \mathrm{m}^{-2} \mathrm{~d}^{-1}\right)$, but there were no differences between these means and those for "normal" conditions $\left(\mathrm{SST}: F_{(2,468)}=2.78, P=0.063\right.$; PP: $\left.F_{(2,453)}=2.60, P=0.075\right)$ (table 1).

At regional scale, mean $\mathrm{Chl}$ decreased significantly during EN relative to "normal" conditions at the entrance (from 0.44 to $0.30 \mathrm{mg} \mathrm{m}^{-3} ; F_{(2,154)}=19.55, P=0.000$ ), in the central gulf (from 0.90 to $0.72 \mathrm{mg} \mathrm{m}^{-3} ; F_{(2,154)}=1.90, P=$ 0.000 ), and in the MIR (from 1.56 to $1.20 \mathrm{mg} \mathrm{m}^{-3} ; F_{(2,154)}=$ $5.04, P=0.007)$. Under EN conditions, PP was significantly reduced at the entrance (from 0.93 to $0.75 \mathrm{~g} \mathrm{C} \mathrm{m}^{-2} \mathrm{~d}^{-1}$; $\left.F_{(2,149)}=5.81, P=0.003\right)$ but not in the central gulf $\left(F_{(2,149)}=\right.$ $0.39, P=0.679)$ or the $\operatorname{MIR}\left(F_{(2,149)}=1.10, P=0.336\right)$. The increase in $\mathrm{Chl}$ and $\mathrm{PP}$ during $\mathrm{LN}$ was only significant at the entrance, with mean values of $0.68 \mathrm{mg} \mathrm{m}^{-3}\left(F_{(2,154)}=19.55\right.$, $P=0.000)$ and $1.11 \mathrm{~g} \mathrm{C} \mathrm{m}^{-2} \mathrm{~d}^{-1}\left(F_{(2,149)}=5.81, P=0.003\right)$, respectively (table 1 ).

According to the seasonal anomalies, there was a delayed response of 3-6 months in the reestablishment of "normal" phytoplankton activity levels once the EN events had ceased (fig. 2). Phytoplankton response to favorable LN conditions was not immediate and delayed even more if the event had been preceded by an EN event. The effects of LN on Chl and PP were also observed after 3-6 months and extended over a similar period. For the entire Gulf of California, there were no significant differences in the effects of the five EN events comparaciones de los promedios de las variables bajo consideración, entre regiones y entre condiciones, los resultados de las pruebas ANOVA fueron confirmados en todos los casos por los de las pruebas Tukey-Kramer, por lo que a continuación se dan sólo los resultados de ANOVA. Los promedios de Chl para todo el golfo durante eventos EN $\left(0.74 \mathrm{mg} \mathrm{m}^{-3}\right)$ y LN $\left(1.00 \mathrm{mg} \mathrm{m}^{-3}\right)$ fueron estadísticamente diferentes $\left(F_{(2,468)}=7.22, P=0.000\right)$ a los estimados en condiciones "normales" (anomalías de TSM cercanas a cero). El decremento del promedio de Chl para todo el golfo durante EN fue $23 \%$ y durante $\mathrm{LN}$ el incremento fue $4 \%$, respecto a las condiciones "normales". Los promedios de TSM y PP para todo el golfo mostraron diferencias significativas entre eventos EN $\left(25.50^{\circ} \mathrm{C}, 1.45 \mathrm{~g} \mathrm{C} \mathrm{m}^{-2} \mathrm{~d}^{-1}\right)$ y $\mathrm{LN}\left(24.19^{\circ} \mathrm{C}\right.$, $1.50 \mathrm{~g} \mathrm{C} \mathrm{m}^{-2} \mathrm{~d}^{-1}$ ), pero los promedios en estos eventos no mostraron diferencias con los estimados en condiciones "normales" (TSM: $F_{(2,468)}=2.78, P=0.063$; PP: $F_{(2,453)}=$ 2.60, $P=0.075)$ (tabla 1).

En la escala regional, el promedio de Chl se redujo significativamente en eventos EN respecto a condiciones "normales" en la boca (de 0.44 a $0.30 \mathrm{mg} \mathrm{m}^{-3} ; F_{(2,154)}=19.55$, $P=0.000$ ), en el centro (de 0.90 a $0.72 \mathrm{mg} \mathrm{m}^{-3} ; F_{(2,154)}=1.90$, $P=0.000$ ) y en la RIG (de 1.56 a $1.20 \mathrm{mg} \mathrm{m}^{-3} ; F_{(2,154)}=5.04$, $P=0.007)$. La PP, bajo la condición EN, sólo se redujo significativamente en la boca (de 0.93 a $0.75 \mathrm{~g} \mathrm{C} \mathrm{m}^{-2} \mathrm{~d}^{-1} ; F_{(2,149)}=$ $5.81, P=0.003)$ y no así en el centro $\left(F_{(2,149)}=0.39, P=\right.$ $0.679)$ ni en la RIG $\left(F_{(2,149)}=1.10, P=0.336\right)$. El incremento de Chl y PP durante LN sólo fue significativo en la boca, con un valor promedio de $0.68 \mathrm{mg} \mathrm{m}^{-3}\left(F_{(2,154)}=19.55, P=0.000\right)$ y $1.11 \mathrm{~g} \mathrm{C} \mathrm{m}^{-2} \mathrm{~d}^{-1}\left(F_{(2,149)}=5.81, P=0.003\right)$, respectivamente (tabla 1).

De acuerdo con las anomalías estacionales, existió una respuesta desfasada (3-6 meses) para el restablecimiento de los niveles "normales" de la actividad fitoplanctónica una vez que cesaron los eventos EN (fig. 2). La respuesta del fitoplancton ante las condiciones favorables de LN no fue inmediata, más aún si ésta había sido antecedida por un evento EN. Los efectos de LN sobre la Chl y PP también se observaron tras 3-6 meses y se extendieron por un período igual. A escala global, no se encontraron diferencias significativas entre los impactos de los cinco eventos EN que incidieron durante el período de estudio en la TSM $\left(F_{(4,133)}=0.49, P=\right.$ $0.746)$ y $\mathrm{Chl}\left(F_{(4,133)}=0.47, P=0.761\right)$. Sólo el último evento EN (2009-2010) mostró una diferencia positiva significativa de PP $\left(F_{(4,127)}=14.51, P=0.000\right)$ respecto a eventos anteriores. El evento LN 2010 mostró un incremento significativo de la $\operatorname{TSM}\left(F_{(3,119)}=5.84, P=0.000\right)$ respecto a eventos anteriores, y LN 2007-2008 mostró un incremento significativo de Chl $\left(F_{(3,119)}=6.11, P=0.000\right)$ y PP $\left(F_{(3,116)}=4.72, P=\right.$ $0.003)$.

La señal anual de TSM presentó diferencias significativas entre condiciones cálidas (verano y otoño) y frías (invierno y primavera) a escala global $\left(F_{(3,467)}=332.91, P=0.000\right)$ 
Table 1. Annual, interannual, and seasonal means ( \pm standard error) of sea surface temperature (SST), chlorophyll $a$ (Chl), and primary productivity (PP) in three regions and the entire Gulf of California (1997-2010).

Tabla 1. Promedio anual, interanual y estacional ( \pm error estándar) de la temperatura superficial del mar (TSM), clorofila $a$ (Chl) y productividad primaria (PP) en tres regiones y todo el golfo de California (1997-2010).

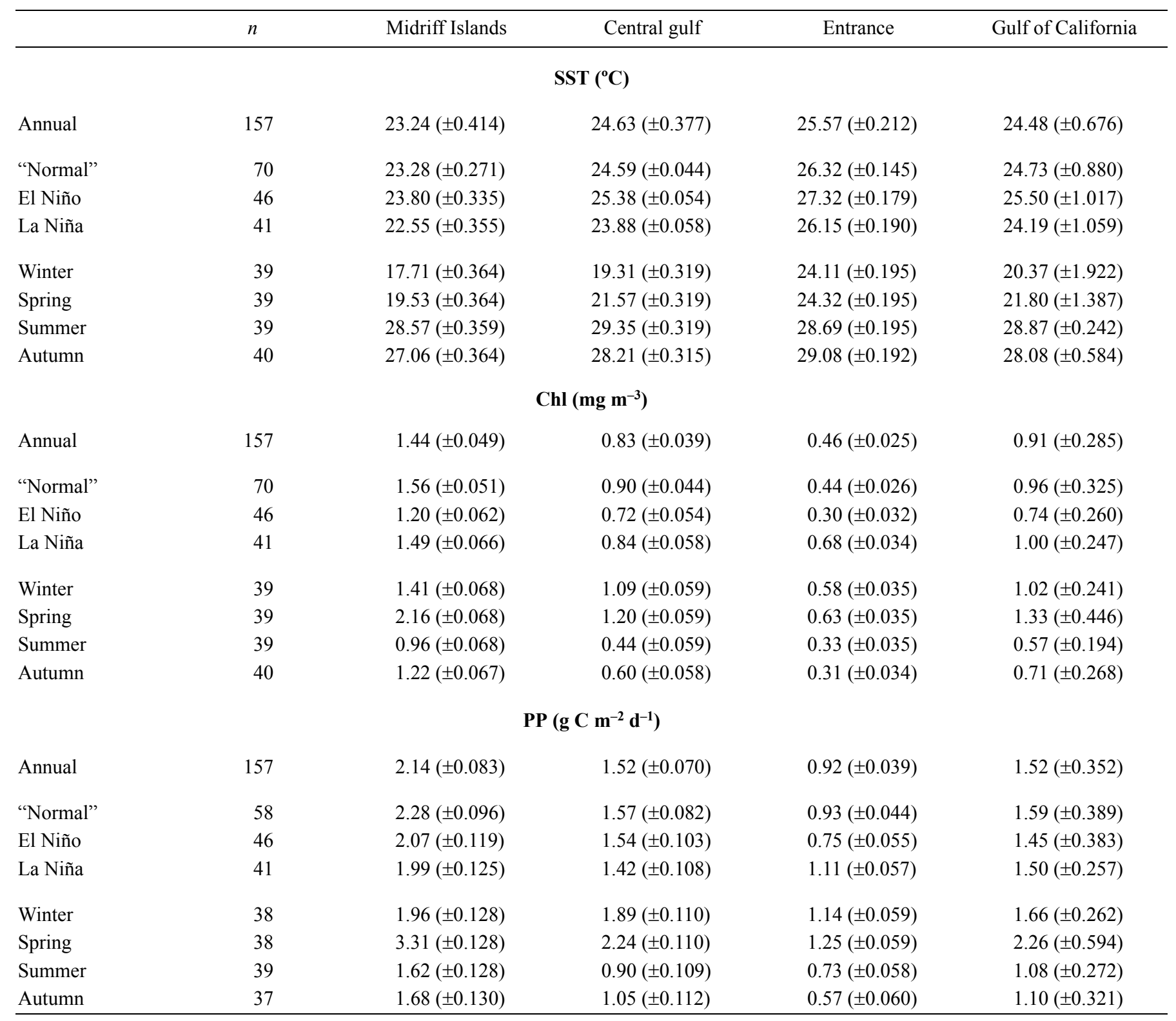

during the study period on $\operatorname{SST}\left(F_{(4,133)}=0.49, P=0.746\right)$ and Chl $\left(F_{(4,133)}=0.47, P=0.761\right)$. Only the last EN event (2009-2010) showed a positive significant difference in PP $\left(F_{(4,127)}=14.51, P=0.000\right)$ relative to the previous events. The $2010 \mathrm{LN}$ showed a significant increase in $\operatorname{SST}\left(F_{(3,119)}=\right.$ 5.84, $P=0.000)$ relative to the previous events, while the 2007-2008 LN showed a significant increase in $\mathrm{Chl}\left(F_{(3,119)}=\right.$ $6.11, P=0.000)$ and PP $\left(F_{(3,116)}=4.72, P=0.003\right)$.

The annual SST signal presented significant differences between warm (summer and autumn) and cold (winter and spring) conditions for the entire gulf $\left(F_{(3,467)}=332.91, P=\right.$ y regional (RIG: $F_{(3,153)}=221.74, P=0.000$; centro: $F_{(3,153)}=$ 228.72, $P=0.000$; boca: $\left.F_{(3,153)}=178.13, P=0.000\right)$ (tabla 1). Bajo condiciones cálidas (frías), la Chl y PP disminuyeron (aumentaron) significativamente en la boca $\left(\mathrm{Chl}: F_{(3,153)}=13.29, P=0.000\right.$; PP: $F_{(3,148)}=23.57, P=$ 0.000) y el centro (Chl: $F_{(3,153)}=39.24, P=0.000$; PP: $\left.F_{(3,148)}=35.69, P=0.000\right)$ del golfo, sin distinción entre verano y otoño (invierno y primavera). Sin embargo, en la RIG la PP de invierno (1.96 $\left.\mathrm{g} \mathrm{C} \mathrm{m}^{-2} \mathrm{~d}^{-1}\right)$ no fue significativamente diferente a la de verano (1.62 $\left.\mathrm{g} \mathrm{C} \mathrm{m}^{-2} \mathrm{~d}^{-1}\right)$ y otoño $\left(1.68 \mathrm{~g} \mathrm{C} \mathrm{m}^{-2} \mathrm{~d}^{-1}\right)\left(F_{(3,148)}=39.85, P=0.000\right)$ (tabla 1). 


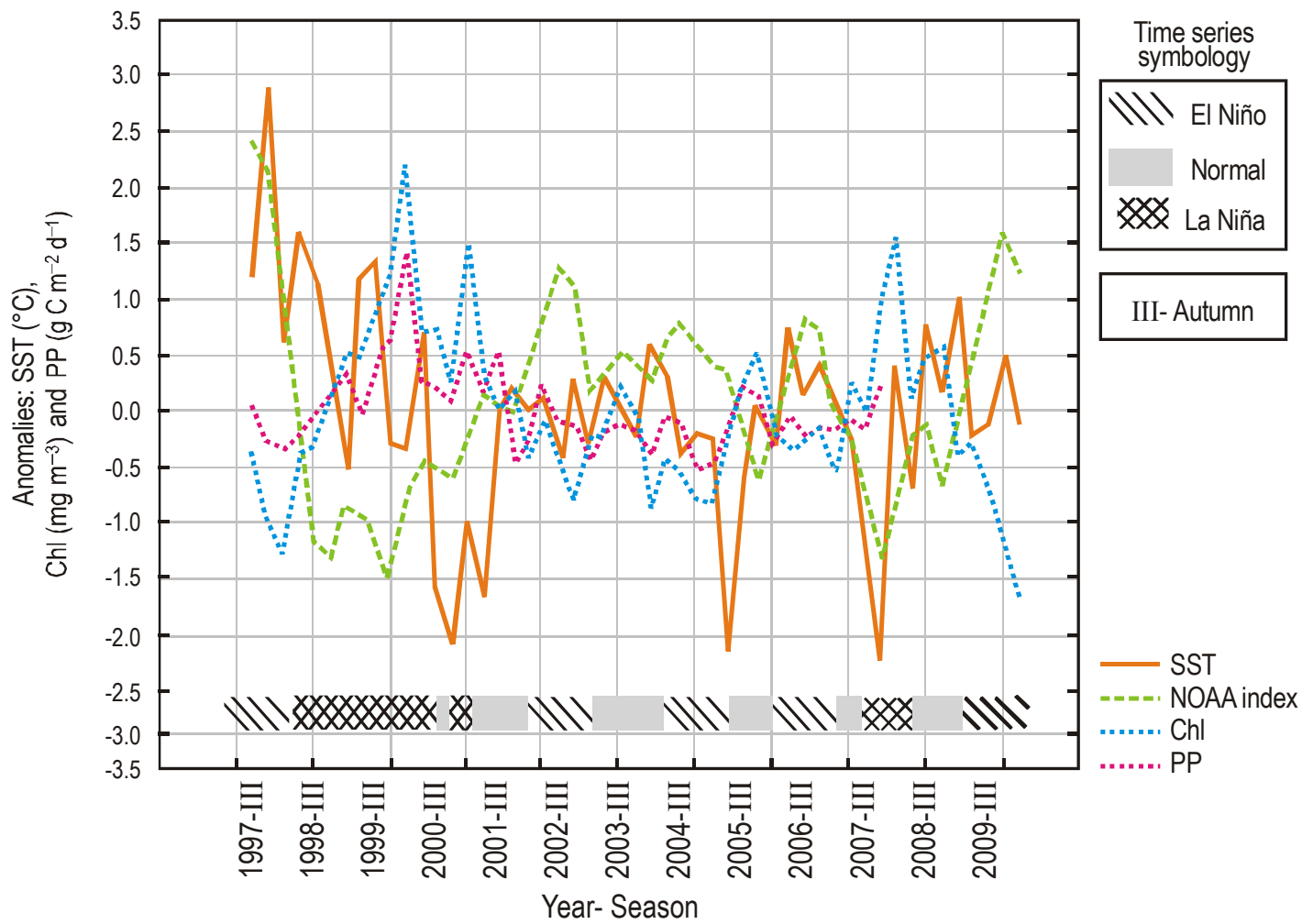

Figure 2. Temporal sea surface temperature (SST), surface chlorophyll $a(\mathrm{Chl})$, and primary productivity (PP) anomalies in the Gulf of California, and SST anomaly index of the NOAA Climate Prediction Center.

Figura 2. Serie temporal de anomalías de la temperatura superficial del mar (SST), clorofila $a(\mathrm{Chl})$ superficial y productividad primaria (PP) en el golfo de California, y el índice de anomalías de la SST del Centro de Predicción del Clima de la NOAA.

0.000 ) and at regional scale (MIR: $F_{(3,153)}=221.74, P=$ 0.000 ; central gulf: $F_{(3,153)}=228.72, P=0.000$; entrance: $F_{(3,153)}=178.13, P=0.000$ ) (table 1). Under warm (cold) conditions, $\mathrm{Chl}$ and PP decreased (increased) significantly at the entrance $\left(\mathrm{Chl}: F_{(3,153)}=13.29, P=0.000\right.$; PP: $F_{(3,148)}=$ 23.57, $P=0.000)$ and in the central gulf $\left(\mathrm{Chl}: F_{(3,153)}=39.24\right.$, $P=0.000$; PP: $\left.F_{(3,148)}=35.69, P=0.000\right)$, with no differences between summer and autumn (winter and spring). In the MIR, however, wintertime PP $\left(1.96 \mathrm{~g} \mathrm{C} \mathrm{m}^{-2} \mathrm{~d}^{-1}\right)$ was not significantly different from that of summer $\left(1.62 \mathrm{~g} \mathrm{C} \mathrm{m}^{-2} \mathrm{~d}^{-1}\right)$ and autumn $\left(1.68 \mathrm{~g} \mathrm{C} \mathrm{m}^{-2} \mathrm{~d}^{-1}\right)\left(F_{(3,148)}=39.85, P=0.000\right)$ (table 1).

The highest annual PP was recorded in the MIR (781 g C $\left.\mathrm{m}^{-2} \mathrm{yr}^{-1}\right)$ and the lowest at the entrance $\left(336 \mathrm{~g} \mathrm{C} \mathrm{m}^{-2} \mathrm{yr}^{-1}\right)$. In the MIR and the central gulf, mean annual $\mathrm{P}_{\mathrm{N}}$ represented more than $60 \%$ of PP. At seasonal scale, the percentage of $P_{N}$ for these two regions was particularly high in winter and spring. At the entrance, mean annual $\mathrm{P}_{\mathrm{N}}$ represented $40 \%$ of $\mathrm{PP}$, and was particularly low ( $\leq 25 \%$ of PP) in summer and autumn. The results for the entire gulf indicated that in winter, spring, summer and autumn, the percentage of PP attributable to $\mathrm{P}_{\mathrm{N}}$ was $67 \%, 76 \%, 49 \%$, and $50 \%$, respectively (table 2).
La PP anual más alta se presentó en la RIG $\left(781 \mathrm{~g} \mathrm{C} \mathrm{m}^{-2}\right.$ año ${ }^{-1}$ y la más baja en la boca $\left(336 \mathrm{~g} \mathrm{C} \mathrm{m}^{-2}\right.$ año $\left.{ }^{-1}\right)$. En la RIG $y$ en el centro del golfo, el porcentaje anual promedio de $\mathrm{P}_{\mathrm{N}}$ fue mayor que el $60 \%$ de PP. A escala estacional, el porcentaje de $\mathrm{P}_{\mathrm{N}}$ para estas dos regiones fue particularmente alto en invierno y primavera. En la región de la boca, el porcentaje anual promedio de $\mathrm{P}_{\mathrm{N}}$ fue $40 \%$ de PP, y fue particularmente bajo ( $\leq 25 \%$ de PP) en verano y otoño. Los resultados globales sugieren que en invierno, primavera, verano y otoño, el porcentaje promedio de PP atribuible a $\mathrm{P}_{\mathrm{N}}$ fue $67 \%, 76 \%$, $49 \%$ y $50 \%$, respectivamente (tabla 2 ).

\section{DISCUSIÓN}

El promedio anual de la TSM presentó un gradiente con incremento de sur a norte, en concordancia con lo descrito por Soto-Mardones et al. (1999). La RIG promedió la TSM más baja debido a la intensa dinámica de mezcla por fenómenos asociados a las mareas que ocurre en esta región (Álvarez-Borrego y Schwartzlose 1979). Esta dinámica física propicia un flujo de nutrientes a la zona eufótica que promueve una alta biomasa y productividad del fitoplancton (Gilbert y Allen 1943, Álvarez-Borrego y Lara-Lara 1991), observándose en esta región los valores promedio más altos 


\section{DISCUSSION}

The resulting south-to-north SST gradient concurs with that described by Soto-Mardones et al. (1999). The MIR averaged the lowest SST due to the intense tide-related mixing that occurs in this area (Álvarez-Borrego and Schwartzlose 1979). This physical process produces a flow of nutrients to the euphotic zone that promotes high phytoplankton biomass and productivity (Gilbert and Allen 1943, Álvarez-Borrego and Lara-Lara 1991), and the highest Chl and PP values were recorded in this region. The intermediate SST, Chl, and PP values were recorded in the central gulf. The mouth, with the highest SST, is characterized by a wellstratified water column, mainly in summer, which restricts the input of nutrients to the euphotic zone and limits photosynthetic activity; hence, the Chl and PP values were lower at the entrance than in the other gulf regions. The characteristics of the gulf mouth (Valdez-Holguín 1986, Álvarez-Borrego and Lara-Lara 1991, Santamaría-del-Ángel 1994a) are determined by its direct communication with the Tropical Pacific Ocean, which allows the inflow of warm, oligotrophic Equatorial Surface Water (Álvarez-Borrego and Schwartzlose 1979, Torres-Orozco 1993). de Chl y PP. La región central promedió valores de TSM, Chl y PP intermedios. La boca del golfo, con la TSM más alta, está caracterizada por una fuerte estratificación de la columna de agua, principalmente en verano, lo cual restringe el aporte de nutrientes a la zona eufótica y limita la actividad fotosintética; por ende, en esta región se produjeron los valores promedio más bajos de Chl y PP que en las otras regiones del golfo. Las características de la región de la boca (Valdez-Holguín 1986, Álvarez-Borrego y Lara-Lara 1991, Santamaría-del-Ángel 1994a) están determinadas por la comunicación directa del golfo con el océano Pacífico Tropical, que permite la penetración del Agua Superficial Ecuatorial, cálida y oligotrófica (Álvarez-Borrego y Schwartzlose 1979, Torres-Orozco 1993).

En condiciones de "verano", la masa de Agua Superficial Ecuatorial invade la región central del golfo de California (Álvarez-Borrego y Schwartzlose 1979, Torres-Orozco 1993), y los vientos dominantes del sureste, cálidos y húmedos, (Roden 1964) no rompen la estratificación de la columna de agua, por lo que la TSM estacional no fue estadísticamente diferente entre la región central y la boca en verano y otoño. En invierno y primavera, esta masa de agua invade solamente la región de la boca (Álvarez-Borrego

Table 2. Annual and seasonal new production $\left(\mathrm{P}_{\mathrm{N}}\right)$ and regenerated production $\left(\mathrm{P}_{\mathrm{R}}\right)$ in three regions and the entire Gulf of California (1997-2010). * Surface area covered.

Tabla 2. Producción nueva $\left(\mathrm{P}_{\mathrm{N}}\right)$ y regenerada $\left(\mathrm{P}_{\mathrm{R}}\right)$, anual y estacional, en tres regiones y en todo el golfo de California (1997-2010). * Área superficial cubierta.

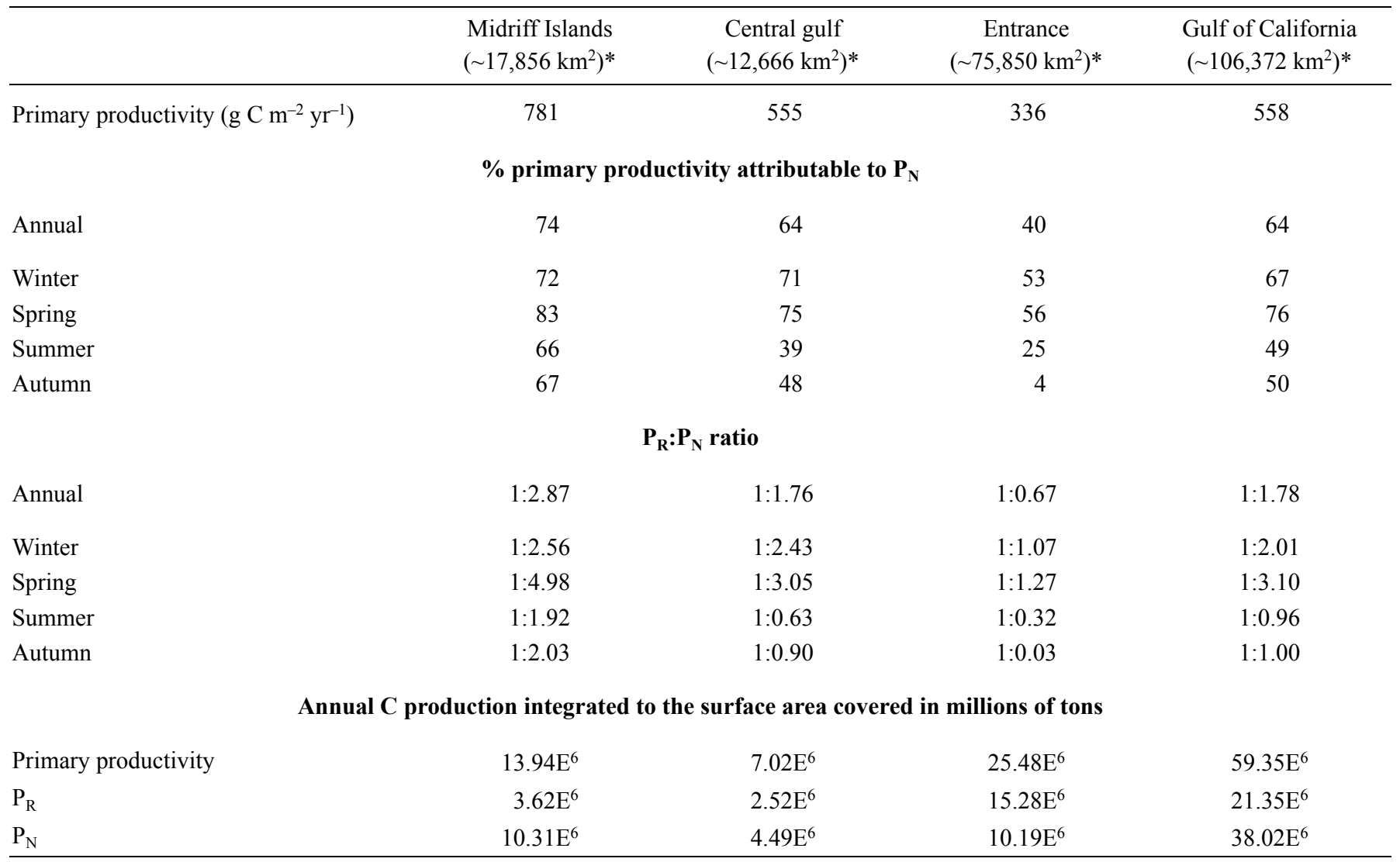


Under "summer" conditions, Equatorial Surface Water invades the central Gulf of California, and the dominant warm and humid southeasterly winds (Roden 1964) do not break the water column stratification, so seasonal SST was not statistically different between the central gulf and the entrance in summer and autumn. In winter and spring, this water mass only invades the mouth region (Álvarez-Borrego 2010), and northwesterly winds generate upwelling all along the east gulf coast and southward to Colima (López-Sandoval et al. 2009), with greater intensity in the central region (Roden 1964); hence, mean Chl and PP values are higher in the central gulf than at the entrance. In the MIR, the mean $\mathrm{Chl}$ and PP values were highest in spring and decreased by $\sim 40 \%$ under "summer" conditions. In winter, water column stratification is weak, and the effects of tide- and windinduced mixing are more intense as phytoplankton are maintained in a turbulent environment (unable to become conditioned to high irradiances); in summer and autumn, the increase in SST reduces the input of nutrients to the euphotic zone and the Chl and PP values are lower. Santamaría-delÁngel et al. (1994a) reported similar variation patterns.

Under EN conditions, mean Chl in the Gulf of California was lower than under "normal" and LN conditions due to the sinking of the thermocline, which inhibits the input of nutrients to surface waters by upwelling (Glantz 2006); however, PP was not statistically different from that under "normal" conditions, possibly because of the inflow of tropical water that transports smaller cells (Lara-Lara and Valdez-Holguín 1988) capable of flourishing with low nutrient levels (Mann and Lazier 2006). Under LN conditions, Chl was significantly different than under "normal" conditions, but PP did not show significant differences relative to these latter conditions. This is probably because, under LN conditions, the physical dynamics of the gulf, with greater turbulence in the water column, likely favors the dominance of large cells, and $\mathrm{Chl}$ and PP values increase because the physiological conditioning and maximum photosynthetic capacity of phytoplankton are deterred, as reported by Álvarez-Borrego and Gaxiola-Castro (1988) and Valdez-Holguín et al. (1999).

The EN signal significantly reduced $\mathrm{Chl}$ in all the regions relative to other conditions. PP was not statistically different in any of the regions under $\mathrm{EN}$ conditions relative to "normal" conditions, but at the entrance there was a significant decrease relative to $\mathrm{LN}$ conditions. Among regions, the latitudinal gradient under "normal" conditions did not change, but under LN conditions the mean values for the central gulf and entrance were similar because of an increase in Chl and PP at the mouth. In the central gulf, physical processes and local effects (presence of filaments and cold-water jets from the northern gulf, tidal currents, wind and upwelling, as described by Ripa and Marinone (1989)) buffer and mask the effects of EN (Valdez-Holguín and Lara-Lara 1987). The relatively oligotrophic conditions at the entrance with low PP values (Álvarez-Borrego and Lara-Lara 1991) did not decline under EN conditions, even though the effects
2010), y los vientos del noroeste producen surgencias costeras en toda la costa este del golfo hasta la costa de Colima (sur del golfo) (López-Sandoval et al. 2009), con mayor intensidad en la región central (Roden 1964); esto genera un gradiente con valores promedio de Chl y PP mayores en la región central que en la boca. En la RIG, los promedios de Chl y PP presentaron los valores más altos en primavera, y se redujeron hasta en un $\sim 40 \%$ con condiciones de "verano". En invierno, la estratificación es débil, por lo que la mezcla por fenómenos asociados a las mareas y los vientos tiene efectos más intensos que mantienen al fitoplancton en un medio turbulento (no se logra acondicionar a irradiancias elevadas); en verano y otoño, el incremento en la TSM reduce el aporte de nutrientes a la zona eufótica y los valores de Chl y PP son menores. Santamaría-del-Ángel et al. (1994a) registraron patrones de variación similares a nuestros resultados.

Bajo condiciones EN, la Chl en el golfo de California fue menor que bajo condiciones "normales" y LN, debido a un hundimiento de la termoclina que inhibe la entrada de nutrientes a las aguas superficiales por efecto de surgencia (Glantz 2006); sin embargo, la PP no fue estadísticamente diferente de la de condiciones "normales", posiblemente por la entrada de agua de origen tropical que transporta células de menor tamaño (Lara-Lara y Valdez-Holguín 1988) que pueden florecer con concentraciones bajas de nutrientes (Mann y Lazier 2006). Bajo condiciones LN, la Chl mostró diferencias significativas con la $\mathrm{Chl}$ bajo condiciones "normales", pero la PP no mostró diferencias significativas respecto a estas últimas condiciones. Lo anterior posiblemente se debe a que, bajo condiciones $\mathrm{LN}$, la dinámica física del golfo (con mayor turbulencia en la columna de agua) debe favorecer la dominancia de células grandes, manifestándose en un incremento en la concentración de Chl y valores de PP similares, ya que no permite que el fitoplancton se acondicione fisiológicamente y que presente valores correspondientes a su máxima capacidad fotosintética, como lo registrado por Álvarez-Borrego y Gaxiola-Castro (1988) y Valdez-Holguín et al. (1999).

La señal EN redujo significativamente la $\mathrm{Chl}$ en todas las regiones respecto a las otras condiciones. La PP no fue estadísticamente diferente en ninguna de las regiones bajo la condición EN, respecto a condiciones "normales", pero en la región de la boca sí hubo disminuciones significativas con relación a la de LN. Entre regiones, el gradiente latitudinal bajo condiciones "normales" no cambió en eventos EN, pero en eventos $\mathrm{LN}$ los valores promedio de la región central y de la boca fueron similares, debido a un incremento en la Chl y $\mathrm{PP}$ en la región de la boca. En la región central, los procesos físicos y efectos locales (presencia de filamentos y chorros de agua fría provenientes del norte del golfo, corrientes de marea, vientos y surgencias, como lo describen Ripa y Marinone (1989)) amortiguan los efectos de EN enmascarándolos (Valdez-Holguín y Lara-Lara 1987). Las condiciones relativamente oligotróficas de la boca con valores de PP bajos (Álvarez-Borrego y Lara-Lara 1991) no disminuyeron 
of EN on Chl are greater at the mouth than in the rest of the gulf (Valdez-Holguín and Lara-Lara 1987, Santamaría-delÁngel et al. 1994b, Hidalgo-González and Álvarez-Borrego 2004). PP integrated per unit area at the entrance can be high even though surface PP is low, due to the greater depth of the euphotic zone at the mouth than in the inner gulf (ValdezHolguín and Lara-Lara 1987). In the MIR and central gulf, the high surface PP values, in comparison with those for the entrance, are sustained by the input of nutrients from outside the euphotic zone. In these regions, nitrogen is made available to phytoplankton by divergence and mixing processes that transport it to the surface, with lesser input of reduced forms excreted by bacteria and zooplankton.

Our findings indicate that the effects of $\mathrm{LN}$ on $\mathrm{Chl}$ and PP were more evident at the mouth. It has been suggested that phytoplankton abundance is lower at the mouth than in the inner gulf (Gilbert and Allen 1943) and that $75 \%$ of carbon assimilation is associated with nanophytoplankton (Berman 1975, Álvarez-Borrego and Lara-Lara 1991). Small cells generally dominate in stable environments (Lara-Lara et al. 1993), favoring a more accelerated growth of phytoplankton populations as an adaptive response to the environment (Sun et al. 2007); hence, the availability of nutrients promoted by LN duplicates photosynthetic activity at the mouth. In the central gulf and MIR, phytoplankton (mainly microphytoplankton) abundance is generally higher, of the order of millions of cells per liter in Guaymas Basin and $>100,000$ cells per liter in the northern gulf (Gilbert and Allen 1943) during "winter" conditions, and greater nutrient input associated with LN events does not significantly increase photosynthetic activity. The effect of LN on PP is slightly attenuated during spring in the central gulf and MIR due to the physical dynamics; intense wind- and tide-induced vertical mixing turbulently transports phytoplankton across the water column, upward and downward, and prevents phytoplankton conditioning to high irradiances, as suggested by several authors for the 1998-1999 LN (Valdez-Holguín et al. 1999, Espinosa-Carreón and Valdez-Holguín 2007). Grazing is another factor that possibly attenuates the effect of LN on $\mathrm{PP}$, as it does not allow photosynthetic rate to increase due to a decrease in productive biomass (García-Pámanes and LaraLara 2001).

The maximum "winter" Chl values of Santamaría-delÁngel et al. (1994a) were much higher for sites on the eastern side than on the western side of the Gulf of California, indicating that the phytoplankton biomass variability caused by EN may be greater on the eastern side. Herrera-Cervantes et al. (2010) removed the annual and semiannual signals from the SeaWifs 1997-2006 Chl data for orthogonal empirical function (OEF) analysis. They found that the spatial distribution of $\mathrm{OEF}_{1}$ (explaining $44 \%$ of the total interannual variance of $\mathrm{Chl}$ ) was more variable on the eastern side of the gulf, its variability decreasing from the mouth towards Tiburón Island, and less variable in the MIR, the northern gulf, and along the west coast. The present study was not bajo la condición EN, a pesar de que los efectos de EN sobre la Chl son mayores en la boca que en el resto del golfo (Valdez-Holguín y Lara-Lara 1987, Santamaría-del-Ángel et al. 1994b, Hidalgo-González y Álvarez-Borrego 2004). La PP integrada por unidad de área en la boca puede ser grande aunque la PP superficial sea baja, debido a la mayor profundidad de la zona eufótica en la boca que en el interior del golfo (Valdez-Holguín y Lara-Lara 1987). En la RIG y el centro del golfo, los valores altos de PP superficial, en comparación con los de la boca, están soportados por el aporte de nutrientes desde fuera de la zona eufótica. En estas regiones, el nitrógeno es puesto a disposición del fitoplancton mediante los procesos de divergencia y de mezcla, que lo transportan a la superficie, con un aporte menor de formas reducidas procedentes de la excreción de zooplancton y bacterias.

Los resultados de este estudio indicaron que los efectos de LN sobre la Chl y la PP fueron más evidentes en la boca del golfo. Se ha sugerido que la abundancia de fitoplancton es menor en la boca del golfo que en el interior (Gilbert y Allen 1943) y que el $75 \%$ de la asimilación de carbono está asociada al nanofitoplancton (Berman 1975, ÁlvarezBorrego y Lara-Lara 1991). Las células pequeñas generalmente dominan en ambientes estables (Lara-Lara et al. 1993) y ello favorece un crecimiento más acelerado de las poblaciones de fitoplancton como respuesta adaptativa al medio (Sun et al. 2007), de tal manera que la disponibilidad de nutrientes propiciada por LN duplica la actividad fotosintética en la boca. En el centro y la RIG, la abundancia de fitoplancton (predominantemente microfitoplancton) es generalmente mayor, del orden de millones de células por litro en la cuenca de Guaymas y >100,000 células por litro en la región norte (Gilbert y Allen 1943) durante condiciones de "invierno", y un mayor aporte de nutrientes asociado a los eventos LN no incrementa significativamente la actividad fotosintética. El efecto de LN en la PP es un tanto atenuado en primavera en la región central y RIG debido a la alta dinámica física; la mezcla vertical intensa por vientos y mareas transporta por turbulencia al fitoplancton a lo largo de la columna de agua, hacia arriba y abajo, y no permite el acondicionamiento del mismo a irradiancias altas, como lo sugirieron diversos autores para LN 1998-1999 (Valdez-Holguín et al. 1999, Espinosa-Carreón y Valdez-Holguín 2007). Otro aspecto que posiblemente atenúa el efecto de LN en la PP es el pastoreo, que no permite que se incremente la tasa fotosintética por una disminución de la biomasa productiva (García-Pámanes y Lara-Lara 2001).

Los valores máximos de Chl de "invierno" que registra Santamaría-Del-Ángel et al. (1994a) fueron mucho mayores para localidades de la parte oriental del golfo que para las de la parte occidental, y esto indica que la mayor variabilidad de biomasa fitoplanctónica causada por EN podría encontrarse en el lado oriental del golfo. Herrera-Cervantes et al. (2010) removieron las señales anual y semianual de los datos de Chl de 1997-2006 del SeaWiFS para realizar un análisis de funciones empíricas ortogonales (FEO). Estos últimos autores 
designed to compare mean $\mathrm{Chl}$ values on the eastern and western sides of the Gulf of California.

Our PP values are similar to those reported by Lara-Lara et al. $(1984,1993)$ and Álvarez-Borrego et al. (1987), which for the most part are in situ estimates. Mean annual PP estimated in this study for all the gulf was $558 \mathrm{~g} \mathrm{C} \mathrm{m}^{-2} \mathrm{yr}^{-1}$ (ranging from 242 to $777 \mathrm{~g} \mathrm{C} \mathrm{m}^{-2} \mathrm{yr}^{-1}$ ). Lluch-Cota et al. (2004) and Kahru et al. (2004) reported mean values of 477 and $506 \mathrm{~g} \mathrm{C} \mathrm{m}^{-2} \mathrm{yr}^{-1}$, respectively, estimated with the same method as that used here. Hidalgo-González and ÁlvarezBorrego (2004) used satellite data and nonhomogeneous Chl profiles to estimate PP for different gulf regions. The deep chlorophyll maximum is a permanent oceanic feature (Dandonneau 1979) and very common in the Gulf of California, and if it is not considered, PP may be subestimated (Hidalgo-González and Álvarez-Borrego 2001). The mean PP values estimated for all the gulf by HidalgoGonzález and Álvarez-Borrego (2004) were $\sim 1.85 \mathrm{~g} \mathrm{C} \mathrm{m}^{-2}$ $\mathrm{d}^{-1}, \sim 20 \%$ higher than those reported herein. A high percentage $(64 \%)$ of $\mathrm{PP}$ is attributable to the input of new nutrients, especially in the MIR (74\%) and in spring (83\%). At the entrance to the gulf, annual PP is mainly attributable to $P_{R}$ $(60 \%)$, reflecting the oligotrophic conditions of the region. Based on these results, it can be inferred that in the Gulf of California there is a relatively high flux of organic carbon from the surface layer to deep waters and the bottom.

\section{CONCLUSIONS}

The concentrations of $\mathrm{Chl}$ and PP were greater in the MIR. In this region, the effects of EL and LN on Chl and PP were not significant as at the entrance, where EL (LN) significantly decreased (increased) their concentrations.

The variations in Chl and PP did not reveal a decrease (or increase) linked to the intensity of the EN (LN) events that occurred between autumn 1997 and autumn 2010.

Our findings suggest that the response of the $\mathrm{Chl}$ and PP signals to the negative (positive) SST anomalies under interannual EL (LN) conditions was explained by the sequence and alternation of abnormal events with normal periods that allowed the reestablishment of the (positive) Chl $a$ and PP anomalies.

The inferences of $\mathrm{P}_{\mathrm{N}}$ suggest that in the Gulf of California it accounts for slightly more than half of the PP (52-64\%) and $\mathrm{P}_{\mathrm{R}}$ accounts for the rest (48-36\%). In the MIR and central gulf, $\mathrm{P}_{\mathrm{N}}$ exceeds $\mathrm{P}_{\mathrm{R}}$, whereas the opposite occurs at the entrance to the gulf.

\section{ACKNOWLEDGMENTS}

This study was supported by the National Council for Science and Technology (CONACYT, Mexico, grant 84944 to JEVH). The first author acknowledges receipt of a postgraduate scholarship from CONACYT. Satellite data were obtained from the NOAA CoastWatch Program and NESDIS encontraron que el patrón espacial de la $\mathrm{FEO}_{1}$ (que explicó $44 \%$ de la variancia interanual total de Chl) mostró que era más variable en el lado oriental del golfo, con su variabilidad disminuyendo de la región de la boca hacia isla Tiburón, mientras que en la RIG, en el golfo norte, y en toda la costa oeste, el $\mathrm{FEO}_{1}$ fue mucho menos variable. El presente trabajo no fue diseñado para comparar valores promedio de $\mathrm{Chl}$ del lado oriental con los del lado occidental del golfo.

Los valores de PP del presente trabajo son similares a los registrados por Lara-Lara et al. (1984), Álvarez-Borrego et al. (1987) y Lara-Lara et al. (1993), que en su mayoría son estimaciones in situ. La PP promedio anual estimada para todo el golfo en este trabajo fue $558 \mathrm{~g} \mathrm{C} \mathrm{m}^{-2} \mathrm{año}^{-1}$, con un intervalo de 242 a $777 \mathrm{~g} \mathrm{C} \mathrm{m}^{-2}$ año-1. El valor promedio registrado por Lluch-Cota et al. (2004) fue $477 \mathrm{~g} \mathrm{C} \mathrm{m}^{-2}$ año $^{-1}$, y el de Kahru et al. (2004) fue $506 \mathrm{~g} \mathrm{C} \mathrm{m}^{-2}$ año $^{-1}$, cuyas estimaciones se hicieron con el mismo método que el de este trabajo. Hidalgo-González y Álvarez-Borrego (2004) usaron datos de satélite y un modelo con perfiles no homogéneos de Chl para estimar PP para regiones diferentes del golfo. El máxímo profundo de Chl (MPC) es una característica muy persistente en el océano (Dandonneau 1979). El MPC es muy común en el golfo de California y el no considerarlo puede conducir a subestimaciones de PP (Hidalgo-González y Álvarez-Borrego 2001). Los valores promedio de PP para todo el golfo estimados por Hidalgo-González y ÁlvarezBorrego (2004) ( $\left.\sim 1.85 \mathrm{~g} \mathrm{C} \mathrm{m}^{-2} \mathrm{~d}^{-1}\right)$ son $\sim 20 \%$ más altos que los registrados en este trabajo. Un alto porcentaje (64\%) de la PP es atribuible al aporte de nutrientes nuevos, particularmente en la RIG (74\%) y en la estación de primavera (83\%). En la boca del golfo la PP anual es principalmente atribuible a la $\mathrm{P}_{\mathrm{R}}(60 \%)$, reflejando las condiciones oligotróficas de la región. Con base en estos resultados se puede inferir que en el golfo de California existe un flujo relativamente elevado de carbono orgánico de la capa superficial hacia aguas profundas y el fondo.

\section{CONCLUSIONES}

La concentración de Chl y PP fue mayor en la RIG. En esta región, los efectos de EN y LN sobre la Chl y PP no fueron significativos como en la boca, región en donde EN (LN) disminuyó (incrementó) significativamente la concentración de Chl y PP.

Las variaciones de Chl y PP no evidenciaron un decremento (o aumento) que estuviera en relación con la intensidad de los eventos EN (LN) que se presentaron de otoño de 1997 a otoño de 2010.

Los resultados del estudio sugieren que la velocidad de respuesta de la señal de Chl y PP a las anomalías negativas (positivas) de temperatura superficial ante las condiciones interanuales EN (LN) fue explicada por la secuencia y alternancia de eventos anormales con períodos normales que permitieron el restablecimiento de las anomalías (positivas) de Chl $a$ y PP. 
Office of Satellite Data Processing and Distribution, and NASA's Goddard Space Flight Center and OceanColor Web.

English translation by Christine Harris.

\section{REFERENCES}

Álvarez-Borrego S. 1983. Gulf of California. In: Ketchum BH (ed.), Estuaries and Enclosed Seas, Elsevier, Amsterdam, pp. 427-449.

Álvarez-Borrego S. 2010. Physical, chemical, and biological oceanography of the Gulf of California. In: Brusca GC (ed.), The Gulf of California: Biodiversity and Conservation. University of Arizona Press, Tucson, pp. 24-48.

Álvarez-Borrego S, Schwartzlose RA. 1979. Water masses of the Gulf of California. Cienc. Mar. 6: 43-63. http://dx.doi.org/10.7773/cm.v6i1.350

Álvarez-Borrego S, Gaxiola-Castro G. 1988. Photosynthetic parameters of Northern Gulf of California phytoplankton. Cont. Shelf Res. 8: 37-47. http://dx.doi.org/10.1016/0278-4343(88)90023-4

Álvarez-Borrego S, Lara-Lara JR. 1991. The physical environment and primary productivity on the Gulf of California. In: Dauphin JP, Simoneit B. (eds.), The Gulf and Peninsular Province of the Californias. American Association of Petroleum Geologists, Tulsa, pp. 555-567.

Álvarez-Borrego S, Gaxiola-Castro G, Hernández-Becerril DU. 1987. The relationship between photosynthesis and irradiance for Gulf of California phytoplankton. Pac. Sci. 4: 79-89.

Behrenfeld JM, Falkowski GP. 1997. A consumer's guide to phytoplankton primary productivity models. Limnol. Oceanogr. 42: 1479-1491. http://dx.doi.org/10.4319/lo.1997.42.7.1479

Beier E, Ripa P. 1998. Seasonal gyres in the northern Gulf of California. J. Phys. Oceanogr. 29: 305-311.

Berman T. 1975. Size fractionation of natural aquatic populations associated with autotrophic and heterotrophic carbon uptake. Mar. Biol. 3: 215-220. http://dx.doi.org/10.1007/BF00390925

Carrillo Bibriezca LE, Lavin Peregrina MF, Palacios-Hernandez E. 2002. Seasonal evolution of the geostrophic circulation in the northern Gulf of California. Estuar. Coast. Shelf Sci. 54: $157-173$.

Dajoz R. 2002. Tratado de Ecología. 2nd ed. Mundi-Prensa, Madrid, $600 \mathrm{pp}$.

Dandonneau Y. 1979. Concentrations en chlorophylle dans le Pacifique Tropical Sud-ouest: Comparison avec d'autres Aires Océaniques Tropicales. Oceanol. Acta 4: 23-32.

Dugdale RC, Goering JJ. 1967. Uptake of new and regenerated forms of nitrogen in primary productivity. Limnol. Oceanogr. 12: 196-206. http://dx.doi.org/10.4319/lo.1967.12.2.0196

Espinosa-Carreón LT, Valdez-Holguín JE. 2007. Variabilidad interanual de clorofila en el Golfo de California. Ecol. Aplicada 6: 81-92.

García-Pámanes J, Lara-Lara JR. 2001. Microzooplanton grazing in the Gulf of California. Cienc. Mar. 27: 73-90.

Gilbert JY, Allen WE. 1943. The phytoplankton of the Gulf of California obtained by the E.W. Scripss in 1939 and 1940. J. Mar. Res. 5: 89-110.

Glantz HM. 2006. Forecasting El Niño: Science's gift to the 21st century. In: Babkina MA (ed.), El Niño Overview and Bibliography. NOVA, USA, pp. 29-40.
Las inferencias de $\mathrm{P}_{\mathrm{N}}$ sugirieron que en el golfo de California es poco más de la mitad de PP (52-64\%) y el resto es $P_{R}(48-36 \%)$. En la RIG y la región central, la $P_{N}$ supera a la $P_{R}$, mientras que en la boca ésta última rebasa a la $P_{N}$.

\section{Agradecimientos}

Agradecemos al Consejo Nacional de Ciencia y Tecnología (CONACYT) el apoyo recibido a través del proyecto (no. 84944) otorgado al segundo autor y la beca de posgrado otorgada a la primera autora. Agradecemos al CoastWatch Program y a la NESDIS Office of Satellite Data Processing and Distribution de la NOAA, al Goddard Space Flight Center de la NASA y al OceanColor Web por la información de satélite.

Herrera-Cervantes H, Lluch-Cota SE, Lluch-Cota DB, GutiérrezDe-Velasco-San-Román G, Lluch-Belda D. 2010. ENSO influence on satellite-derived chlorophyll trends in the Gulf of California. Atmósfera 23: 253-262.

Hidalgo-González RM, Álvarez-Borrego S. 2001. Chlorophyll profiles and the water column structure in the Gulf of California. Oceanol. Acta 24: 19-28.

Hidalgo-González RM, Álvarez-Borrego S. 2004. Total and new production in the Gulf of California estimated from ocean color data from the satellite sensor SeaWIFS. Deep-Sea Res. II 51: 739-752.

Kahru M, Marinone SG, Lluch-Cota SE, Parés-Sierra A, Mitchell GB. 2004. Ocean-color variability in the Gulf of California: Scales from days to ENSO. Deep-Sea Res. II 51: 139-146. http://dx.doi.org/10.1016/j.dsr2.2003.04.001

Lara-Lara JR, Valdez-Holguín JE. 1988. Size fraction biomass and primary productivity in the Gulf of California during spring 1984. Cienc. Mar. 14(1): 1-14.

Lara-Lara JR, Valdez-Holguín JE, Jiménez-Pérez C. 1984. Plankton studies in the Gulf of California during the 1982-1983 El Niño. Trop. Ocean Atmos. Newslett. 28: 16-17.

Lara-Lara JR, Millán-Núñez R, Lara-Osorio JL, Bazán-Guzmán C. 1993. Phytoplankton productivity and biomass by size classes in the central Gulf of California during spring 1985. Cienc. Mar. 19(2): 137-154.

Lavín MF, Organista S. 1988. Surface heat flux in the Northern Gulf of California. J. Geophys. Res. 93: 14033-14038. http://dx.doi.org/10.1029/JC093iC11p14033

Lavin Peregrina MF, Marinone Moschetto SGL. 2003. An overview of the physical oceanography of the Gulf of California. In: Velasco Fuentes OU, Sheinbaum J, Ochoa de la Torre JL (eds.), Nonlinear Processes in Geophysical Fluid Dynamics. Kluwer Academic Publishers, pp. 173-204.

Lavín MF, Palacios-Hernández E, Cabrera C. 2003. Sea surface temperature anomalies in the Gulf of California. Geofís. Int. 42: 363-375.

Lluch-Cota SE, Aragón-Noriega EA, Arreguín-Sánchez F, AuriolesGamboa D, Bautista-Romero JJ, Brusca RC, Cervantes-Duarte R, Cortés-Altamirano R, Del-Monte-Luna P, Esquivel-Herrera A, Fernández G, Hendricks M, Hernández-Vázquez S, Karhu M, Lavín M, Lluch-Belda D, Lluch-Cota DB, López-Martínez J, Marinone SG, Nevárez-Martínez MO, Ortega-García S, Palacios-Hernández E, Parés-Sierra A, Ponce-Díaz G, Ramírez 
M, Salinas-Zavala CA, Schwartzlose RA, Sierra-Beltrán AP. 2004. The Gulf of California: Ecosystem view and environmental trends. Marine ecosystems of the North Pacific. North Pacific Marine Science Organization, PICES Special Publication, USA, $1280 \mathrm{pp}$.

López-Sandoval DC, Lara-Lara JR, Álvarez-Borrego S. 2009. Primary production by remote sensing in the region off Cabo Corrientes, Mexico. Hidrobiológica 19: 185-192.

Mann HK, Lazier NR. 2006. Dynamics of Marine Ecosystems: Biological-Physical Interactions in the Oceans. 3rd ed. WileyBlackwell, USA, 489 pp.

Merrifield MA, Winant CD. 1989. Shelf circulation in the Gulf of California: A description of the variability. J. Geophys. Res. 94(C12): 18133-18160. http://dx.doi.org/10.1029/JC094iC12p18133

Morel A, Berthon JF. 1989. Surface pigments, algal biomass profiles, and potential production of the euphotic layer: Relationships reinvestigated in view of remote-sensing applications. Limnol. Oceanogr. 34: 1545-1562.

Ripa P, Marinone SG. 1989. Seasonal variability of temperature, salinity, velocity, vorticity and sealevel in the central Gulf of California, as inferred from historical data. Q. J. R. Meteorol. Soc. 115: 887-913. http://dx.doi.org/10.1002/qj.49711548807

Roden GI. 1964. Oceanographic aspects of the Gulf of California. In: Van Andel TH, Shor GG (eds.), Marine Geology in the Gulf of California. Mem. Am. Assoc. Pet. Geol. 3: 30-58.

Santamaría-del-Ángel E, Álvarez-Borrego S, Muller-Karger FE. 1994a. The 1982-1984 El Niño in the Gulf of California as seen in the coastal zone color scanner imagery. J. Geophys. Res. 99(C4): 7423-7431.

Santamaría-del-Ángel E, Álvarez-Borrego S, Muller-Karger FE. 1994b. Gulf of California biogeographic regions based on coastal zone color scanner imagery. J. Geophys. Res. 99(C4): $7411-7421$.
Santamaría-del-Angel E, Álvarez-Borrego S, Millán-Núñez R, Muller-Karger FE. 1999. Sobre el efecto débil de las surgencias de verano en la biomasa fitoplanctónica del Golfo de California. Rev. Soc. Mex. Hist. Nat. 49: 207-212.

Soto-Mardones LS, Marinone SG, Parés-Sierra A. 1999. Time and spatial variability of sea surface temperature in the Gulf of California. Cienc. Mar. 25: 1-30.

Sun J, Feng Y, Zhang Y, Hutchins D. 2007. Fast microzooplankton grazing on fast-growing, low biomass phytoplankton: A case study in spring in Chesapeake Bay, Delaware Inland Bays and Delaware Bay. Hydrobiologia 589: 127-139. http://dx.doi.org/10.1007/s10750-007-0730-6

Torres-Orozco E. 1993. Análisis volumétrico de las masas de agua del Golfo de California. MSc thesis, Centro de Investigación Científica y de Educación Superior de Ensenada, Baja California, México, $80 \mathrm{pp}$.

Valdez-Holguín JE. 1986. Distribución de la biomasa y productividad del fitoplancton en el golfo de California durante el evento de El Niño 1983-1983. MSc thesis, Centro de Investigación Científica y de Educación Superior de Ensenada, Baja California, México, 92 pp.

Valdez-Holguín JE, Lara-Lara JR. 1987. Primary productivity in the Gulf of California: Effects of El Niño 1982-1983 event. Cienc. Mar. 13(2): 34-50.

Valdez-Holguín JE, Álvarez-Borrego S, Trees, CC. 1999. Seasonal and spatial Characterization of the Gulf of California phytoplankton photosynthetic parameters. Cienc. Mar. 25: 445-467.

White AE, Prhal FG, Letelier RM, Popp BN. 2007. Summer surface waters in the Gulf of California: Prime habitat for biological $\mathrm{N}_{2}$ fixation. Global Biogeochem. Cycles 21(GB2017-10.1029): $1-11$. http://dx.doi.org/10.1029/2006GB002779

Zeitzschel B. 1969. Primary productivity in the Gulf of California. Mar. Biol. 3: 201-207. http://dx.doi.org/10.1007/BF00360952

Received September 2012, received in revised form April 2013, accepted May 2013. 